\title{
Archéopages
}

Archéopages

Archéologie et société

40 | 04-07/2014

Villages

\section{Le village, entre continuité et discontinuité. Débat}

Propos recueillis par Catherine Chauveau

Isabelle Catteddu, Claude Raynaud, Jean-Pierre Le Goff et Catherine Chauveau

\section{CpenEdition}

\section{Journals}

Édition électronique

URL : https://journals.openedition.org/archeopages/643

DOI : $10.4000 /$ archeopages. 643

ISSN : 2269-9872

Éditeur

INRAP - Institut national de recherches archéologiques préventives

Édition imprimée

Date de publication : 1 juin 2015

Pagination : 148-153

ISSN : 1622-8545

Référence électronique

Isabelle Catteddu, Claude Raynaud, Jean-Pierre Le Goff et Catherine Chauveau, « Le village, entre continuité et discontinuité. Débat », Archéopages [En ligne], 40 | 04-07/2014, mis en ligne le 01 juillet 2016, consulté le 23 janvier 2022. URL : http://journals.openedition.org/archeopages/643; DOI : https://doi.org/10.4000/archeopages.643 


\section{village,

\section{discontinuité}

Coincé entre la ville et la campagne, le village peine à trouver sa place dans les typologies de l'habitat. Coincée entre les effets de sources textuelles, matérielles, conceptuelles, et la force des modèles qu'elles génèrent, l'archéologie peine à le définir. Au-delà des interrogations portant sur sa naissance et sur sa mort se pose particulièrement à nous celle sur les dynamiques de longévité et de renouvellement.

\section{8}

\section{Isabelle Catteddu}

est ingénieur chargé de recherche à l'Inrap et membre de l'équipe « Archéologies environnementales » de l'UMR 7041 Archéologie et Sciences de l'Antiquité. Parmi ses dernières publications : avec P. De Vingo et A. Nissen Jaubert (dir.), Theme 2. Archaeology and rural landscape: rural settlements in their natural economical and social environment, On the Road again. L'Europe en Mouvement, $4^{\text {th }}$ International Congress of Medieval and Modern archaeology, De Ferrari, Gênes-Genova, 2011; avec F. Carre, F. Gentili et al., « Fouilles d'églises rurales du haut Moyen Age dans le Nord de la France », in D. Paris-Poulain, D. Istria et S. Nardi Combescure (dir.), Les premiers temps chrétiens dans le territoire de la France Actuelle, Presses Universitaires de Rennes, Rennes, 2009, p. 201-222 ; sous sa direction, Les habitats carolingiens de Montours et de la Chapelle-SaintAubert(Ille-et-Vilaine), Éd. de la Maison des Sciences de l'Homme, DAF n89, Paris, 2001.

\section{Claude Raynaud}

est archéologue au CNRS, dans l'équipe «Territoires et sociétés de l'Antiquité au Moyen Âge » de l'UMR 5140 Archéologie des Sociétés Méditerranéennes. Parmi ses dernières publications : sous sa direction, Archéologie d'un village languedocien.

Lunel-Viel (Hérault) du I Ir au X VIII siècle, Éd. de l'Association pour le développement de l'archéologie en Languedoc-Roussillon, coll. Monographies d'archéologie méditerranéenne, Lattes, 2007;

«Typologie archéologique de l'habitat rural » et « La dynamique spatiotemporelle de l'habitat rural galloromain » in F. Favory, J.-L. Fiches et S. Van der Leeuw, Archéologie et systèmes socioenvironnementaux. Études multiscalaires sur la vallée du Rhône dans le programme Archaeomedes, Monographies du CRA, 27, p. 201-238, 301-322, 2003.

\section{Jean-Pierre Le Goff}

est sociologue au CNRS, au sein du laboratoire Institutions et Dynamiques historiques de l'économie et de la société (UMR 8533). Ses principaux axes de recherche sont les nouvelles conflictualités et les reconfigurations sociales et culturelles qu'elles entraînent. Il a publié notamment: La fin du village. Une histoire française, Gallimard, Paris, 2012 ; La France morcelée, Gallimard, Paris, 2008.
Propos recueillis par Catherine Chauveau. Merci à Christiane Descombin 


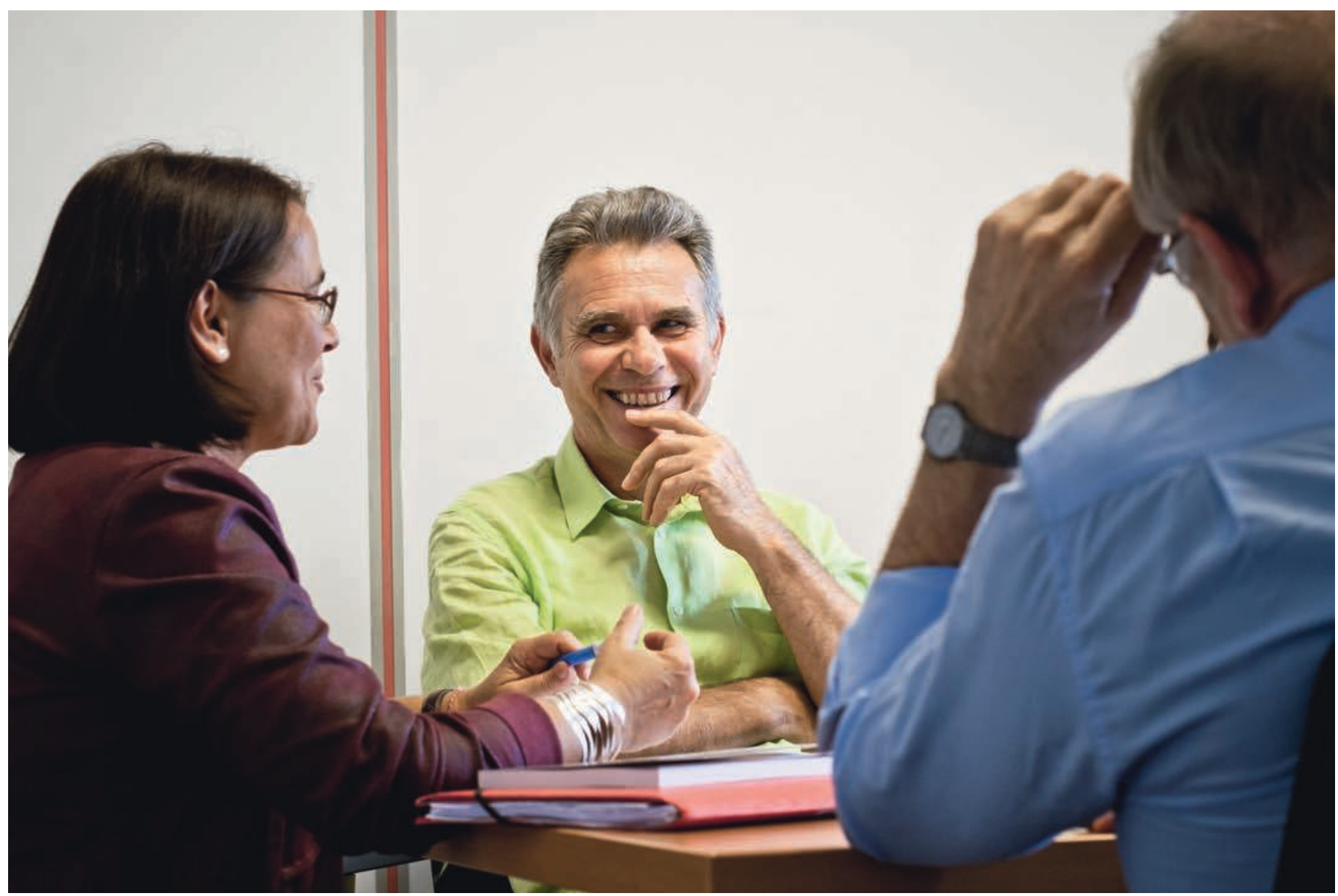

\section{La question n'est plus de débattre de l’objet "village médiéval" mais de s'intéresser à la dynamique du réseau d'habitat. Isabelle Catteddu}

Jean-Pierre Le Goff Le village est l'un des lieux, l'un des milieux, où la question de la reconfiguration sociale et culturelle se pose. Ces dernières décennies, de l'immédiat après-guerre à aujourd'hui, ont connu diverses étapes de modernisation, qui ont été autant de bouleversements pour les populations villageoises françaises. Les sociologues se sont beaucoup intéressés à ce phénomène. Je m’inscris dans la lignée de deux ouvrages majeurs. Le premier est Un Village dans le Vaucluse, de Laurence Wylie', qui décrit le village de Roussillon dans ses aspects extrêmement provençaux que je retrouve totalement dans le récit des anciens du village que j'ai étudié, Cadenet. On est dans les années 1950, terriblement marquées par l'avant-guerre, encore dans le village républicain de la $\mathrm{II}^{\mathrm{e}}$ République. Le deuxième livre est celui d'Edgar Morin, Commune en France. La métamorphose de Plodémet ${ }^{2}$. C'est une autre région de France, dans la période historique qui suit, c'est-à-dire en plein bouleversement des 30 Glorieuses.
On y voit des agriculteurs, engagés avec leurs syndicats d'origine catholique, dans une dynamique de transformation de l'agriculture. Mais qu'est devenu tout cela ensuite ? Le sujet a été abordé, notamment dans l'Histoire de la France rurale dirigée par Georges Duby et Armand Wallon ${ }^{\mathbf{3}}$, car la transformation du village sur une séquence de temps très limitée a marqué profondément l'imaginaire et continue de le marquer. Repensons aux affiches de campagne de Mitterrand. En 1965, il pose dans un paysage de campagne, au fond duquel fument des cheminées d'usine, et devant une ligne à haute tension, avec comme slogan : «Un président jeune pour une France moderne ». En 1981, le paysage d'arrière-plan est celui d'un village, autour de l'église, et le slogan : « La force tranquille». Mais que s'est-il passé ? J'entendais les anciens villageois de Cadenet plaisanter sur le fait qu'ils étaient une espèce en voie de disparition (ils sont les derniers représentants des catégories 
sociales basées sur le travail manuel qui ont façonné la France : les paysans, les ouvriers, les artisans) tandis que ce village ne cessait de s'accroître. Le problème profond est cette discontinuité socioculturelle dans une continuité géographique et historique. De quoi partez-vous pour aborder ces questions?

Claude Raynaud De nouveaux paradigmes, élaborés peu à peu depuis les années 1980. Lorsque les constructions des premiers lotissements ont commencé à LunelViel, en 1979, on a trouvé des fragments de tuiles, de tessons... On attendait une villa comme cela avait été écrit et répété depuis le $19^{\mathrm{e}}$ siècle. On a trouvé une agglomération (bâtiments publics, thermes, voierie, carrefour aménagé, domus) perdurant $\mathrm{du} \mathrm{I}^{\mathrm{er}}$ siècle jusqu'à la fin de l'époque romaine, ainsi que des traces de réoccupation, de transformation, de modification de la trame d'habitat et de la culture matérielle jusquà l'époque moderne. Je l'ai interprété comme étant le premier village gallo-romain, malgré les sarcasmes des spécialistes d'alors, historiens et architectes classiques, qui en parlaient comme de «machins» bricolés. Leur scepticisme correspondait bien à une méconnaissance du sujet: pour eux, il n'y avait d'habitats groupés à l'époque romaine qu'en ville. Dans la tradition historiographique de l'Antiquité, en effet, en dehors de la ville n'existaient que les habitats dispersés, c'est-à-dire les domaines des grands aristocrates qui gèrent la ville et sinon, c'était de l'innommé, un vaste rien. Mais ayant été à l'école de Robert Ferras et de Roger Brunet ${ }^{4}$ à Montpellier, j'avais une définition géographique du village. Et je me suis rapproché de Philippe Leveau qui avait accepté cette vision différente de l'urbanité romaine. À Lunel, on avait des moellons collés, certes, mais maçonnés à la romaine ; on avait du marbre, de petits bouts et non canoniques certes, mais venant de toute la Méditerranée... Il y avait donc une richesse et un statut social que l'on ne trouvait pas dans les fermes voisines qu'on fouillait parallèlement. C'était un échelon intermédiaire entre la ville et la campagne qui n'était pas connu. Alors, du côté des antiquisants, on a repensé le phénomène urbain à la campagne et du côté des médiévistes, on a établi la chronologie de l'habitat groupé.

Isabelle Catteddu Ce qui a été une vraie révolution tant nous étions encombrés, paralysés, en tant que médiévistes du premier Moyen Âge, par la question du village. On ne pouvait même pas prononcer le mot! Dans la définition qu'en avait donnée Robert Fossier ${ }^{5}$, la naissance du village ne s'était enclenchée qu'autour de ce fameux An mil, période de la polarisation des populations autour d'un élément fixateur : l'église, le cimetière, le lieu de pouvoir... Tout ce qui était antérieur était défini comme instable, dispersé, dans un contexte d'une agriculture faible et de paysages peu structurés. Le premier Moyen Âge était comme le négatif de la vision flatteuse du second Moyen Âge bien établie à cette époque. Pourtant, à la même période, dans les autres pays d'Europe, les chercheurs n'étaient pas bloqués dans ce modèle. C'est un litige francofrançais. Mais grâce à l'archéologie préventive à partir des années 1990, on a découvert un très grand nombre de sites d'habitats des époques mérovingiennes et carolingiennes, dont certains très structurés qui collaient mal avec la définition établie. Il a fallu ce changement d'échelle spatiale et temporelle pour regarder ces sites autrement, pouvoir les étudier en lien avec leurs terroirs... et se débarrasser de la vieille définition du village. CR Et il a fallu convaincre nos collègues que la continuité d'occupation d'un même lieu ne voulait pas dire archaïsme, conservation, continuité forcenée mais correspondait plutôt à toute une série d'avatars : une forme d'habitat groupé avait donné lieu à une autre forme d'habitat au même endroit et que les fondations de ces villages successifs étaient inextricablement associées. Et on a démontré que cette continuité d'occupation ne se confondait pas avec une continuité dans la forme de l'habitat et dans la société villageoise.

IC On est presque étonné devoir argumenter sur la diversité des formes d'habitat dans le passé, et leur imbrication, alors que si l'on regarde la France d'aujourd'hui, du Nord au Sud, ils nous sautent aux yeux.

JPLG J'ai fait ce même constat. Ces dernières décennies, avec le vieillissement de la population, la crise des activités traditionnelles, la décrépitude du bâti... se pose la question de la façon de retrouver une dynamique de vie à l'intérieur du village. Et le pari qui est fait à par beaucoup de maires est de développer les infrastructures pour attirer des populations nouvelles, en général venues de la ville. Cependant, pour bien aborder ces processus de changements dans la continuité, on se doit de distinguer une structure de base et des strates successives. Ce qui revient à se demander ce qu'est la principale caractéristique du village. En sociologie, la démarche est d'établir un idéal-type, c'est-à-dire un modèle qui ne correspond jamais à la réalité telle qu'elle existe mais qui permet un certain nombre de regroupements du matériel empirique. L'idéal-type du village, d'un point de vue sociologique, est un espace géographique où sont regroupées trois caractéristiques : lieu d'habitat, lieu de travail et lieu de loisir ; loisir étant une création moderne, disons le temps de la sociabilité en dehors du travail, auquel il faut associer le temps de la sacralité. La fin du village, selon moi, est la dissociation de ces trois caractéristiques au profit d'un lieu à fonction unique. Dans le cas que j'ai étudié, le village de Cadenet, c'est au profit de la fonction d'habitation résidentielle. Mais il y a aussi d'autres aspects à prendre en compte dans la définition de cet idéal-type : le rapport de plus grande proximité avec la nature, qu'elle soit attrayante ou hostile, et l'ordre de proximité interpersonnelle au sein de la communauté. Ce dernier point attirant particulièrement l'attention des sociologues, on s'en doute. Cela inclut à la fois la capacité à régler de façon autonome ses conflits intérieurs y compris 


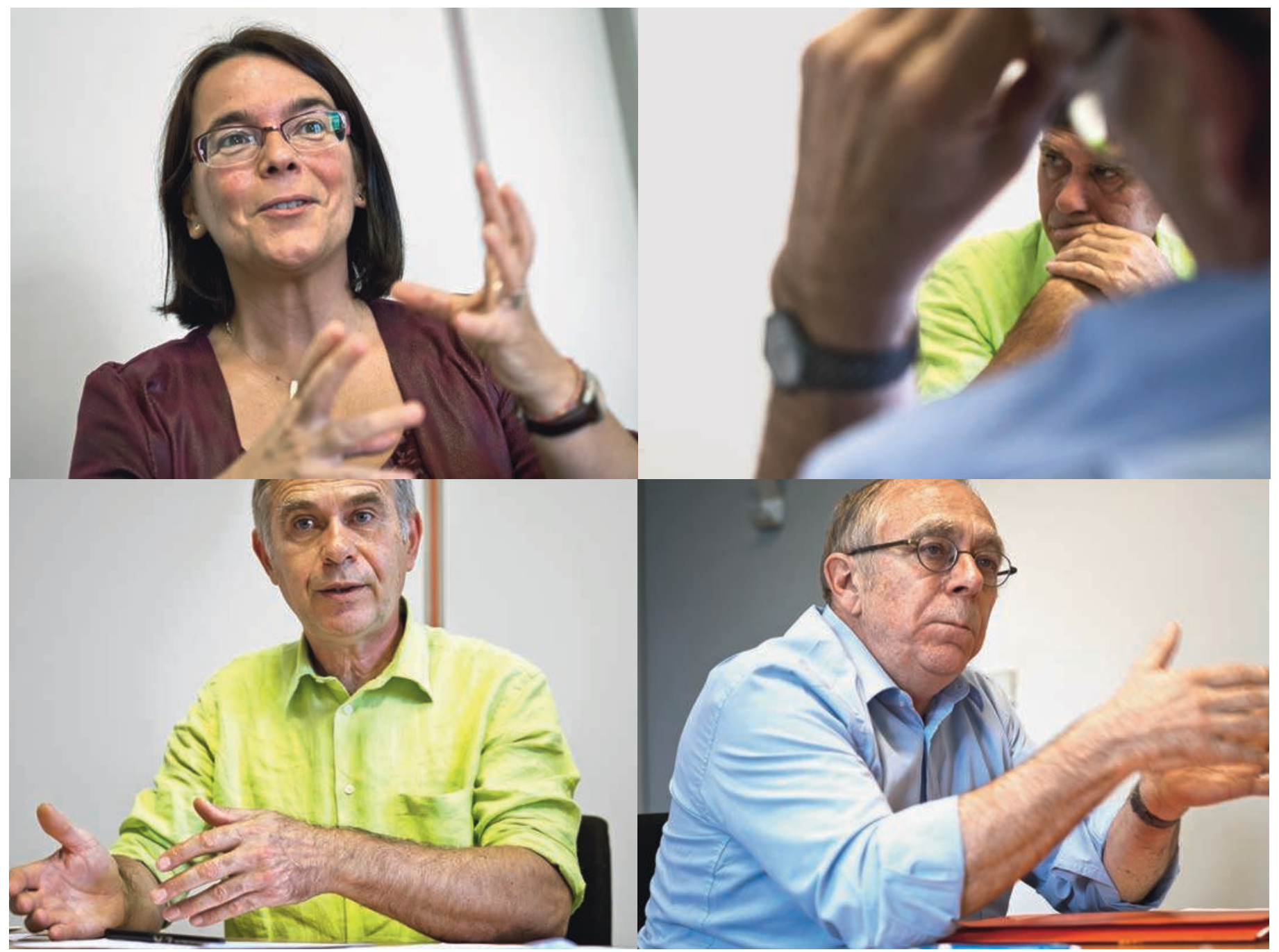

Sur un temps très court, dans un village existe une juxtaposition d'éléments d'uniformisation et de démarcation montrant que la modernité n'a pas complètement opéré. Jean-Pierre Le Goff

avec des instances de religion et un partage de traditions communes malgré des styles de vies tout à fait différents. Cela s'appelle un creuset culturel anthropologique, fait de mémoire, de valeurs et de comportements communs. Et je rajouterai un dernier élément, pas des moindres : l'attachement très fort à ce « chez soi » que l'on retrouve dans des expressions comme « la petite patrie », ou « être pays » ; c'est le Heimat allemand, mot intraduisible qui contient le rapport affectif et le sentiment de familiarité avec un lieu. En bref, les habitants partagent une culture au sens anthropologique du terme. Mais, à l'heure de la mondialisation, il y a ceux qui ont les moyens de choisir leur « chez soi » et ceux qui ne les ont pas. Cela entraine une nouvelle répartition de la population. D’un point de vue sociologique, les nouveaux venus, souvent venus de la ville, gardent les habitudes de mise à distance. Alors que dans l'idéal-type du village, tous se connaissent, se saluent, se composent les uns par rapport aux autres, avec la fin du village, les gens s'ignorent, s'évitent. Dans ce bouleversement, la place et le rôle des modes de consommation ont joué un rôle central dans cette mutation : facilité des transports, télévision, centres commerciaux... S'ajoute à cela la reconfiguration sociale. Dans un village, on avait des catégories principales majoritaires nées d'un rapport à la nature ou d'un rapport à une activité, qui fait que la communauté partage une certaine conception de la condition 
humaine, ce qui renforce la proximité. Quand arrivent des catégories sociales complètement différentes (aujourd'hui, ce sont des retraités, des enseignants, des techniciens,

des professions libérales, des artistes...), il est ardu de coexister alors que l'on a plus d'histoire, de sentiment d'appartenance et de traditions partagées. Ce qui fait que vous avez de nouveaux types de fracture, non seulement en terme économico-social, différence de revenus, mais en termes de mentalité et de culture. Enfin, d'un point de vue urbanistique, il y a un étalement du village à partir de l'ancien noyau ; les lotissements poussent dans des zones périphériques, parfois jusquà sembler s'agréger à un village voisin. Quel serait votre équivalent d'un idéal-type ? Quelle est la réalité matérielle que vous associez à ce mot village? CR Les historiens n'avaient pas conscience que le village pouvait exister avant que les textes n'en parlent. La contradiction apportée par l'archéologie a été de démêler des formes d'habitat groupé dont les textes ne parlaient pas. Ces premières mentions écrites sont de 1096, alors que ce que nous constatons est que ces formes d'habitat groupé existent déjà depuis de nombreux siècles. Ce qui les caractérise est : des bâtiments non résidentiels nécessitant l'intervention de la collectivité ; des habitations familiales ; une organisation autour d'un système de communication ; une place, au sens physique du terme, pour se retrouver et échanger, repère spatial au centre d'un territoire. C'est cette notion de territoire avec celle du partage des ressources qui sont essentielles pour nous.

IC Pour ce qui est du haut Moyen Âge, et du Moyen Âge plus généralement, le premier constat est une grande diversité d'habitats groupés. Le village avec château et église n'est pas l'habitat exclusif du Moyen Age, tout comme la villa n'est pas celui de l'Antiquité. Distinguer les formes d'habitat, on y arrive, les nommer est moins évident et consensuel et on se perd entre la ferme, le groupe de fermes, le hameau, l'habitat groupé, le village.... Tous ces termes-là ne sont pas encore bien définis. Et il ne faut pas les figer trop vite. Car cette diversité nous a obligé à mettre beaucoup plus de nuances dans la description de ces occupations et à se lancer dans de nouvelles réflexions. Par exemple, on a longtemps eu l'impression d'une disparité entre le nord et le sud de la Loire - pour faire simple - avec notamment, la fixation des habitas groupés plus précoce au sud ; mais ces différences sont en train de s'amenuiser au fur et à mesure de l'accroissement du corpus. Par contre, ce qui est constant, c'est la forte relation entre ces habitats groupés, leur terroir et les pratiques agricoles et environnementales. Le voilà le facteur de fixation que l'on voulait comprendre à tout prix! L'autre constante est la forte dynamique de ces habitats groupés : changement de formes, de fonction, de polarités... Se distinguent aussi parfois, très tôt, des secteurs spécialisés et des structures de mise en commun comme les zones de stockage. À Châteaugiron, par exemple, petite ville médiévale à côté de Rennes, on a une continuité d'occupation qui démarre au Néolithique. Dès le IV ${ }^{\mathrm{e}}$ siècle avant notre ère, l'espace agricole est organisé autour de deux fermes par des réseaux de fossé et des chemins. Ensuite, on est au croisement de deux voies romaines très importantes. Et à partir du vi ${ }^{\mathrm{e}}$ siècle l'occupation se densifie selon une organisation à la manière d'un hameau et même d'un lotissement: quadrillage de petites parcelles, reliées par des chemins, multiplication des unités d'habitation, zones dédiées à certaines activités. La ville de Châteaugiron est créée au XI ${ }^{\mathrm{e}}$ siècle, avec son château, mais un peu plus loin, tandis que cette zone d'habitat redevient totalement rurale.

JPLG Les critères de la vie du village sont bien évidemment en rapport à une temporalité longue et à la tradition, et on se rencontre bien sur l'importance de cette notion d'ancrage dans un territoire et celle du lieu de rencontre. Mais un des éléments clé du bouleversement, c'est la temporalité courte. Comment pouvez-vous la percevoir? J'ajoute une autre question : je considère les éléments matériels d'uniformisation actuels (télé, ordinateur, réseaux, produits de consommation...), comme ne permettant pas de rendre compte des démarcations, des divergences. Alors comment les éléments matériels pris en général pourraientils rendre compte des déterminants structuraux de la condition humaine (le rapport à la vie et à la mort, à la nature, au travail, aux alliances...)? J'ai l'impression que vous travaillez sur des choses bien réelles tout en se posant la question des besoins fondamentaux des hommes. Les objets et déterminants environnementaux peuvent être semblables alors que ce qui a amené des sociétés à les choisir peut relever d'éléments très divers. C'est bien ce qui fait le sens de la culture ou de la civilisation.

CR En archéologie, on n'est pas trop gêné pour parler de culture ; mais on parle de culture matérielle. Et nos recherches sont faites de mise en évidence des distinctions, rencontres, partages de diverses cultures. Mais il faut se garder de la tendance à l'ethnicisme. Les archéologues sont parfaitement conscients que les populations se renouvellent, se mêlent, se transforment. Mais ils sont parfois gênés dans leurs analyses par des approches tendancieuses, des repères antérieurs basés sur les races, les ethnies... Prenons l'exemple d'une nécropole fouillée en Roussillon près de Perpignan, dont j'ai fait l'historiographie : en 1938-39, l'archéologue y voyait une population romaine en cours d'évolution, mais lors de la publication en 1942-43, il s'agissait de peuples germaniques et en 1947, finalement, c'était des Romains. JPLG Le terme culture est sans cesse sujet de querelles à n'en plus finir, c'est bien vrai! Le problème sur une réalité qui est éminemment complexe, est qu'on ne peut en maîtriser la totalité. 

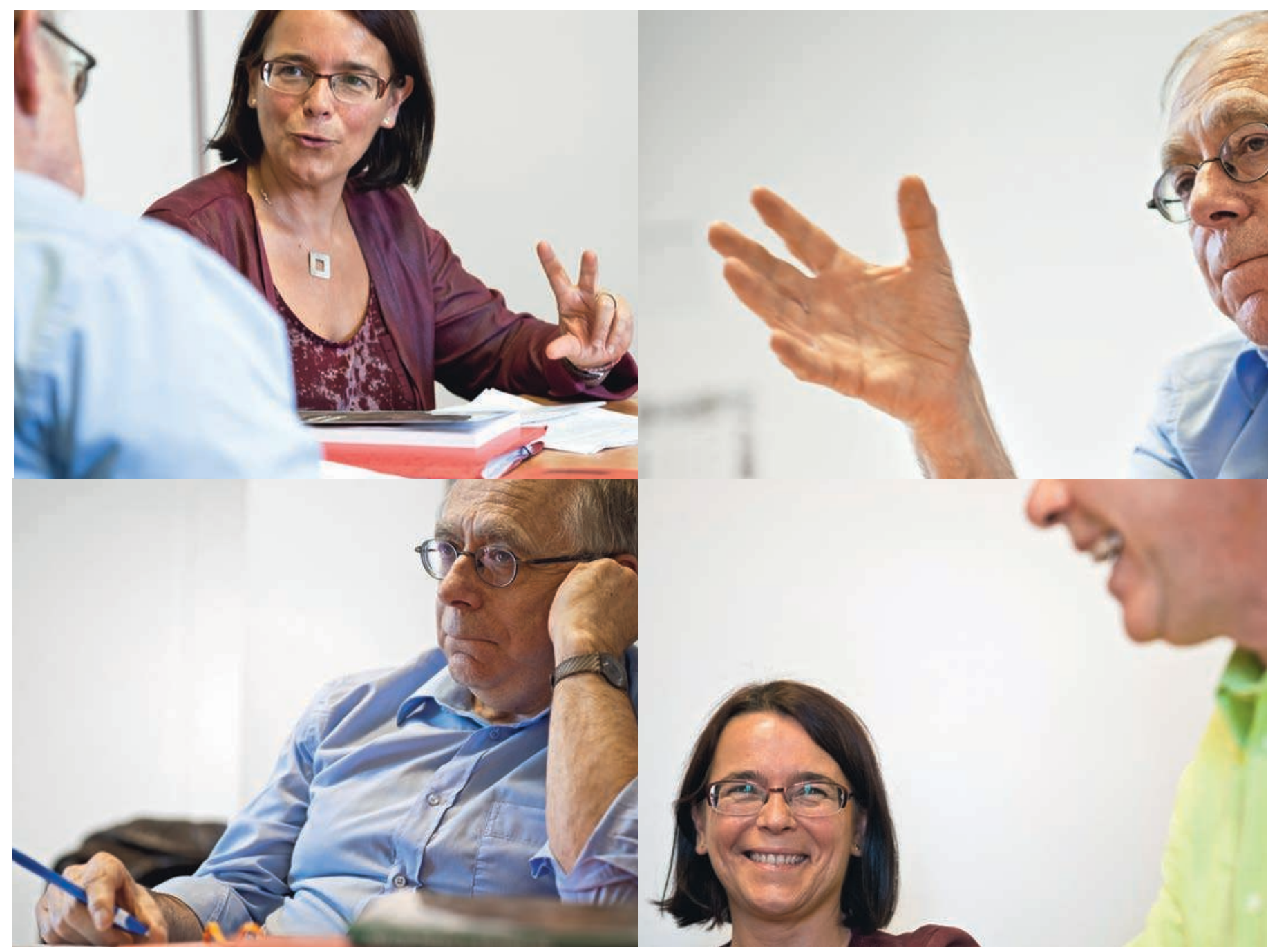

\section{J'ai mis l'accent sur les naissances successives du village de Lunel pour m'opposer à l'idée générique de la naissance du village. Claude Raynaud}

Nous avons chacun des découpes qui montrent une partie du phénomène et tous une démarche inductive, et finalement c'est comme cela que notre compréhension progresse.

CR Si, avant de fouiller un site, on se base sur les évidences émises par la génération précédente de chercheurs, on ne va trouver que des sites qui entrent dans leur nomenclature. Si on n'a pas trouvé de villages pendant des décennies, c'est que l'on cherchait des villae, des lieux de culte... Quand naissent les villages? Je ne peux pas répondre. Dans l'étude que je conduis dans la région de Montpellier, j'ai identifié quatre temps successifs de naissance (début de l'époque romaine, fin de l'Antiquité, haut Moyen Age, autour du XI ${ }^{\mathrm{e}}$ siècle) au gré des dispersions ou recentrages de la population, de l'époque romaine $\mathrm{au} \mathrm{XI}^{\mathrm{e}}$ siècle, mais je suis sûr qu'il y en a d'autres. Et, on voit que d'un village à l'autre, les quatre cas se juxtaposent. Est-ce que les choses sont vraiment synchrones? Je ne le pense pas. Il y a un ensemble de paramètres que l'on a tendance à considérer comme autant d'arrêts sur image alors qu'ils sont, en fait, plus situés dans un continuum. Et ce sont ces processus qui importent. On a besoin en archéologie de remplacer l'histoire des modèles par l'histoire des processus. 